\title{
Lack of association between the risk of prostate cancer and vitamin D receptor Bsm I polymorphism: a meta-analysis of 27 published studies
}

This article was published in the following Dove Press journal:

Cancer Management and Research

\author{
Shaosan Kangl,* \\ Yansheng Zhao 2,* \\ Lei Wang' \\ Jian Liu' \\ Xi Chen' \\ Xiaofeng Liu $^{3}$ \\ Zhijie Shi ${ }^{4}$ \\ Weixing Gao' \\ Fenghong $\mathrm{CaO}^{\prime}$
}

'Department of Urology, North China University of Science and Technology Affiliated Hospital, Tangshan 063000, People's Republic of China; ${ }^{2}$ Department of Imaging, Kailuan General Hospital, Tangshan 063000, People's Republic of China; ${ }^{3}$ Department of Surgery, Laoting Traditional Chinese Medicine Hospital, Tangshan 063600, People's Republic of China; ${ }^{4}$ Department of Urology, Tangshan Gongren Hospital, Tangshan 063000, People's Republic of China

*These authors contributed equally to this work

Correspondence: Shaosan Kang Department of Urology, North China University of Science and Technology Affiliated Hospital, Tangshan, People's Republic of China

Email kangshaosan@I63.com
Background: The association between vitamin D receptor gene Bsm I (rs1544410) polymorphism and prostate cancer ( $\mathrm{PCa}$ ) risk has been investigated by numerous previous studies, which yielded inconsistent results. We conducted this meta-analysis to derive a relatively precise description of this association.

Methods: All studies published up to December 2017 were identified via a systematic search of PubMed, Embase, and China National Knowledge Infrastructure databases. Pooled odds ratios (ORs) with their 95\% confidence intervals (CIs) were estimated to describe the strength of the relationship between $B s m I$ and PCa risk.

Results: In this meta-analysis, 27 studies with 9,993 cases and 9,345 controls were included. The pooled results revealed that Bsm I polymorphism was not associated with PCa risk in the overall analysis. Moreover, no significant relationship was found in the subgroup analyses by ethnicities, genotyping methods, Hardy-Weinberg equilibrium status, and Gleason score. In the stratified analysis by the source of controls and clinical stages, controls of benign prostatic hyperplasia (BPH) seemed to be in the particular groups in which the association of $\mathrm{PCa}$ risk with Bsm I polymorphism was significant ( $\mathrm{Bb}$ vs. bb: $\mathrm{OR}=0.643,95 \% \mathrm{CI}=0.436-0.949$, $p=0.026$; $\mathrm{BB} / \mathrm{Bb}$ vs. bb: $\mathrm{OR}=0.627,95 \% \mathrm{CI}=0.411-0.954, p=0.029 ; \mathrm{B}$ vs. $\mathrm{b}: \mathrm{OR}=0.715,95 \%$ $\mathrm{CI}=0.530-0.965, p=0.029$ ).

Conclusion: Our results suggest that Bsm I polymorphism is weakly associated with PCa risk, and hence, it cannot be considered as a predictor of the occurrence and development of PCa in clinical practice. Future studies with a larger number of samples are needed to verify our results. Keywords: Bsm I, prostate cancer, vitamin D receptor, polymorphisms, meta-analysis

\section{Introduction}

According to a recent report published in the CA: A Cancer Journal for Clinicians in January, 164,690 new prostate cancer (PCa) cases and 29,430 PCa-related deaths were estimated in Americans in 2015. ${ }^{1} \mathrm{PCa}$ has risen to the first place among new cancer cases, and become the second leading cause of cancer-related deaths in males. ${ }^{1}$ To make matters worse, the global prevalence rate of $\mathrm{PCa}$ is rising rapidly. It is forecasted that by 2030 , the number of newly diagnosed PCa cases and deaths will rise up to more than 1.8 million and 0.5 million, respectively. ${ }^{2}$ Existing evidence suggests that PCa risk might increase due to multiple factors, including aging, genetic factors, pathological changes, diet, hormonal level, as well as ethnicity and environment. ${ }^{3}$ However, the pathophysiological mechanism of PCa remains largely unclear. 
In a laboratory investigation, prostate cell division and growth was reported to be affected by vitamin D. ${ }^{4}$ Thus, low plasma levels of vitamin $\mathrm{D}$ were hypothesized to be one of the important contributors to $\mathrm{PCa} .{ }^{4}$ The clinical trial also found that pre-diagnostic serum levels of vitamin $\mathrm{D}>85 \mathrm{nmol} / \mathrm{L}$ may improve survival in men with $\mathrm{PCa} .{ }^{5}$ The action of vitamin $\mathrm{D}$ is mediated by vitamin $\mathrm{D}$ receptor $(V D R) .{ }^{6} 1,25$-Dihydroxy vitamin $\mathrm{D} 3\left(1,25(\mathrm{OH})_{2} \mathrm{D}_{3}\right)$, which is one of the active forms of vitamin $\mathrm{D}$, would combine with $V D R$ to form a heterodimer complex. Subsequently, the complex binds to vitamin $\mathrm{D}$ response element inducing reduced transcriptional levels of many genes which then stimulates tumor cell growth and differentiation. ${ }^{7,8}$

In recent years, the association between PCa risk and some single-nucleotide polymorphisms of $V D R$ gene has become the focus of research attention. ${ }^{9}$ We also conducted a meta-analysis on Taq I and Fok I polymorphisms and their relationships with $\mathrm{PCa}$ risk. ${ }^{7} \mathrm{Bsm} I$ polymorphism (rs1544410) is one of the most frequently researched variants. It is a restriction site located in intron 8 of $V D R$ gene, which does not affect the amino acid sequence during VDR protein expression. ${ }^{10}$ However, mutations in the intron region might be able to lower the stability of mRNA and affect the mRNA levels. Numerous research has revealed that Bsm I mutation might play a significant role in the development or progression of $\mathrm{PCa} .{ }^{11-14}$ However, some other studies do not support this association. ${ }^{15-18}$ These results are inconsistent and worth further exploration. In addition, previous meta-analyse ${ }^{10,19-22}$ seemed to be out of date due to availability of new data. ${ }^{3,9,14,23,24}$ Therefore, we performed a new meta-analysis with the aim of obtaining more accurate and updated results.

\section{Methods}

\section{Literature retrieval strategy}

PubMed, Embase, and China National Knowledge Infrastructure $(\mathrm{CNKI})$ electronic databases were searched for eligible studies published till December 2017. The terms “VDR/ vitamin D receptor", "prostate cancer/tumor/carcinoma", and "polymorphism/mutation/variant" were used for searching titles or abstracts. Full search expressions were "vitamin D receptor [Title/Abstract] AND ((polymorphism [Title/ Abstract] OR mutation [Title/Abstract]) OR variant [Title/ Abstract]) AND prostate cancer [Title/Abstract]" for PubMed, “"vitamin d receptor':ab,ti AND 'polymorphism':ab,ti AND 'prostate cancer':ab,ti” for Embase, and "vitamin D receptor AND polymorphism AND prostate cancer" in Chinese for CNKI. In addition, we read the original or review reports carefully and searched manually for more eligible literature based on their references.

\section{Study selection}

Candidate studies were evaluated by two authors independently (Lei Wang and Jian Liu) for the following inclusion criteria: (1) studies in nonfamilial case-control or nested case-control design conducted on human beings; (2) studies that assessed the relationship between Bsm I polymorphism and risk or progression of $\mathrm{PCa}$; (3) studies in which the distribution frequency of genotype and allelic profile of participants could be acquired or calculated; (4) studies in which no significant difference was reported between cases and controls in the aspect of baseline characters; (5) studies that scored more than 5 points on the Newcastle-Ottawa Scale (NOS).

\section{Data extraction}

Two investigators (Lei Wang and Jian Liu) collected the following information independently: first author's name, publication year, population information, genotyping methods, the number of participants, genotype and allelic profile, as well as the source of controls. Cases and controls were classified into different subgroups by ethnicity, source of controls, and genotyping method, respectively. The subjects were also divided into group with Gleason score $<7$ and group with Gleason score $\geq 7$ by pathological grade, and localized group and aggressive group by clinical stages, respectively. Any controversial content was discussed and evaluated by a third reviewer (Yansheng Zhao) to reach an agreement on all the items.

\section{Statistical analyses}

The heterogeneity was evaluated by using $\chi^{2}$-test based on Cochran's $Q$-test and $I^{2}$ statistics. If $I^{2}>50 \%$ and $p<0.05$, the heterogeneity between studies was significant and the random-effects model was used to combine the values from single studies; ${ }^{25}$ otherwise, in the absence of heterogeneity, the fixed-effects model was chosen. The pooled odds ratios (ORs), together with $95 \%$ confidence intervals (CIs), were calculated to assess the strength of the relationship. The statistical significance of ORs was determined with $Z$-test. Five genetic comparison models were calculated in our analysis, including homozygote model (BB vs. bb), heterozygous model (Bb vs. bb), dominant model (BB vs. $\mathrm{Bb} / \mathrm{bb}$ ), recessive model ( $\mathrm{BB} / \mathrm{Bb}$ vs. $\mathrm{bb}$ ), and allele genetic model (B vs. b allele). Begg's funnel plot and Egger's linear regression were used to evaluate the potential publication 
bias. Sensitivity analysis was performed to evaluate the stability of pooled results. Moreover, the Hardy-Weinberg equilibrium (HWE) status of controls was recalculated with the goodness-of-fit $\chi^{2}$-test; $p<0.05$ indicated that the genotype frequency of controls was not consistent with HWE.

For each outcome, we also conducted subgroup analyses by ethnicity, the source of controls, genotyping method, and clinical stages. $p$-values were two-sided, and $p<0.05$ was considered statistically significant. All analyses were done using the STATA package version 12.0 (Stata Corp, College Station, TX, USA).

\section{Results}

\section{Characteristics of studies}

A total of 87 studies were identified to be potentially related to the topic through our search strategy. Following our inclusion criteria, 27 studies $^{3,9,11-17,23,24,26-41}$ published between the years 1998 and 2017 were finally included to evaluate the association (Figure 1). As shown in Table 1, out of the 27 studies, 25 explored the relationship of PCa risk with Bsm I, and nine were about the association between PCa progression and Bsm I. The number of participants in the case group and control group varied from 28 to 1,034, and 30 to 1,566 , respectively. For all studies, except five, the genotype distribution frequency of Bsm I polymorphism in the control groups conformed to the HWE. All the studies scored more than 5 on the NOS, and were considered to be of high quality (Table 1).

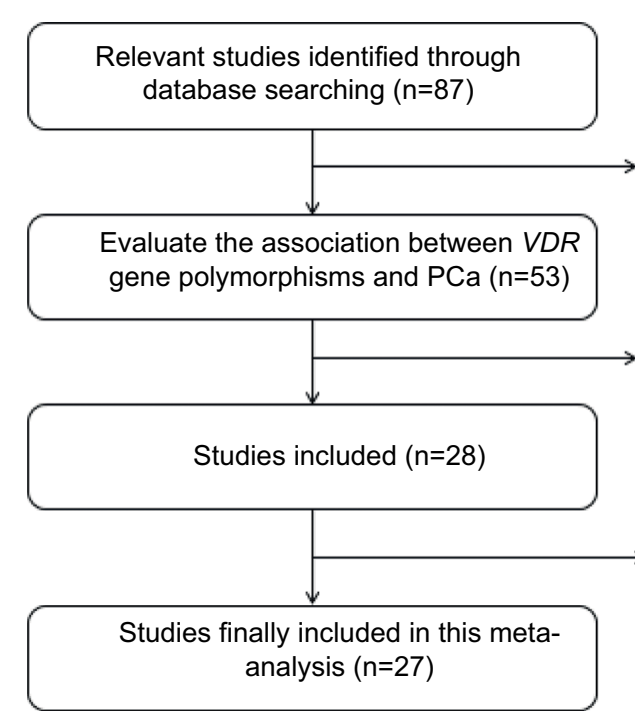

Figure I Flowchart showing the process of selection of the final 27 studies. Abbreviation: $\mathrm{PCa}$, prostate cancer.

\section{Heterogeneity}

Obvious heterogeneity between the studies was found in overall analysis for some genetic comparison models ( $\mathrm{Bb}$ vs. bb: $p=0.000, I^{2}=59.2 \%$; BB/Bb vs. bb: $p=0.000, I^{2}=65.9 \%$; and B vs. b: $p=0.000, I^{2}=65.8 \%$ ) (Tables $2-6$ ). Thus, the random-effects model was chosen for data analysis in these comparison models. Meanwhile, in the recessive model, no heterogeneity was detected (BB vs. $\mathrm{Bb} / \mathrm{bb}: p=0.285$, $I^{2}=12.5 \%$ ), and the fixed-effects model was used. Similar results were found in the subgroup analyses.

\section{Pooled results in terms of PCa risk with Bsm I polymorphism}

The results of the overall analysis obtained by pooling all the 25 studies are shown in Table 2 and Figure 2. These results indicate that $B s m I$ mutation does not increase the risk of $\mathrm{PCa}$ under different comparison models (BB vs. bb: $\mathrm{OR}=0.977,95 \% \mathrm{CI}=0.889-1.074, p=0.634 ; \mathrm{Bb}$ vs. bb: $\mathrm{OR}=0.940,95 \% \mathrm{CI}=0.825-1.072, p=0.357 ; \mathrm{BB} / \mathrm{Bb}$ vs. bb: $\mathrm{OR}=0.951,95 \% \mathrm{CI}=0.832-1.087, p=0.462 ; \mathrm{BB}$ vs. $\mathrm{Bb} /$ bb: $\mathrm{OR}=1.002,95 \% \mathrm{CI}=0.923-1.087, p=0.963$; B vs. $\mathrm{b}$ : $\mathrm{OR}=0.969,95 \% \mathrm{CI}=0.883-1.065, p=0.516$ ) (Table 2).

In the subgroup analyses conducted for a more detailed evaluation of the relationship, the results did not reveal any association by different ethnicities (Table 3 ), different genotyping methods (Table 4), or different HWE statuses of control groups (results not shown).

As shown in Figure 3 and Table 5, in the stratified analysis by the source of control groups, the PCa risk was significantly
Records removed based on titles and abstracts $(n=34)$

Records removed based on full-text articles $(n=25)$

-Without sufficient data extraction, $n=2$ -Not examining Bsm / polymorphism, $\mathrm{n}=22$ -Familial case-control design, $n=1$

Duplicate publication, $n=1$ 
Table I Characteristics and quality assessment of the studies included in this meta-analysis

\begin{tabular}{|c|c|c|c|c|c|c|c|c|}
\hline Author & Year & Country & Ethnicity & $\begin{array}{l}\text { Genotyping } \\
\text { method }\end{array}$ & $\begin{array}{l}\text { Source of } \\
\text { controls }\end{array}$ & Sample size (cases/controls) & HWE & NOS \\
\hline Bai et al ${ }^{11 *}$ & 2009 & $\begin{array}{l}\text { People's Republic } \\
\text { of China }\end{array}$ & Asian & PCR-RFLP & $\mathrm{HB}$ & $122 / 130$ & $\mathrm{Y}$ & 6 \\
\hline Chaimuangraj et al ${ }^{15}$ & 2006 & Thailand & Asian & PCR-RFLP & $\mathrm{HB} / \mathrm{BPH}$ & $28 / 30 / 44$ & $\mathrm{~N} / \mathrm{N}$ & 5 \\
\hline Chen et $\mathrm{a}^{26}$ & 2001 & $\begin{array}{l}\text { People's Republic } \\
\text { of China }\end{array}$ & Asian & PCR-RFLP & $\mathrm{HB}$ & $95 / 103$ & Y & 5 \\
\hline Cheteri et $\mathrm{a}^{27 *}$ & 2004 & USA & Caucasian & PCR-RFLP & PB & $543 / 510$ & $\mathrm{~N}$ & 6 \\
\hline Chokkalingam et al ${ }^{16 *}$ & 2001 & $\begin{array}{l}\text { People's Republic } \\
\text { of China }\end{array}$ & Asian & PCR-RFLP & PB & $161 / 297$ & $N$ & 6 \\
\hline Cicek et a ${ }^{28 *}$ & 2006 & USA & Mixed & PCR-RFLP & PB & $493 / 479$ & Y & 7 \\
\hline El Ezzi et $\mathrm{al}^{24}$ & 2014 & Lebanon & Asian & PCR-RFLP & $\mathrm{BPH}$ & $50 / 68$ & $\mathrm{~N}$ & 5 \\
\hline El Ezzi et $a^{23}$ & 2017 & Lebanon & Asian & PCR-RFLP & PB & $50 / 79$ & $Y$ & 6 \\
\hline Habuchi et al ${ }^{12}$ & 2000 & Japan & Asian & PCR-RFLP & $\mathrm{PB} / \mathrm{BPH}$ & $222 / 326 / 209$ & $Y / Y$ & 8 \\
\hline Hayes et al ${ }^{17}$ & 2005 & Australia & Caucasian & PCR-RFLP & PB & $812 / 713$ & $Y$ & 8 \\
\hline Holick et $\mathrm{a}^{29}$ & 2007 & USA & Caucasian & SNPlex & PB & $590 / 541$ & $Y$ & 8 \\
\hline Holt et a ${ }^{30}$ & 2009 & USA & Mixed & SNPlex & PB & $795 / 767$ & $Y$ & 8 \\
\hline Huang et $\mathrm{al}^{13 *}$ & 2004 & $\begin{array}{l}\text { People's Republic } \\
\text { of China }\end{array}$ & Asian & PCR-RFLP & PB & $160 / 205$ & $\mathrm{~N}$ & 6 \\
\hline Jingwi et $\mathrm{al}^{9}$ & 2015 & USA & African & TaqMan & $\mathrm{HB}$ & $278 / 71$ & $Y$ & 7 \\
\hline Li et $\mathrm{al}^{31}$ & 2007 & USA & Caucasian & PCR-RFLP & PB & $1034 / 1566$ & $Y$ & 8 \\
\hline Liu et $\mathrm{al}^{32}$ & 2003 & $\begin{array}{l}\text { People's Republic } \\
\text { of China }\end{array}$ & Asian & HPLC & PB & $103 / 106$ & $Y$ & 7 \\
\hline Ma et $\mathrm{al}^{33}$ & 1998 & USA & Caucasian & PCR-RFLP & PB & $372 / 591$ & $Y$ & 7 \\
\hline Mikhak et a ${ }^{34}$ & 2007 & USA & Caucasian & TaqMan & PB & $646 / 669$ & $Y$ & 7 \\
\hline Nam et al ${ }^{35}$ & 2003 & Canada & Mixed & PCR-RFLP & $\mathrm{HB} / \mathrm{BPH}$ & $483 / 548 / 256$ & $N / Y$ & 7 \\
\hline Nunes et $\mathrm{al}^{14 *}$ & 2016 & Brazil & Caucasian & PCR-RFLP & $\mathrm{PB} / \mathrm{BPH}$ & $|32 / 169 / 4|$ & $Y / Y$ & 7 \\
\hline Oakley-Girvan et a $\left.\right|^{36}$ & 2004 & USA & Mixed & PCR-RFLP & PB & $345 / 292$ & $Y$ & 7 \\
\hline Oh et $\mathrm{al}^{3}$ & 2014 & South Korea & Asian & SNPlex & $\mathrm{BPH}$ & $272 / 173$ & $Y$ & 6 \\
\hline Onen et $\mathrm{al}^{37}$ & 2008 & Turkey & Caucasian & PCR-RFLP & PB & $133 / 157$ & $Y$ & 7 \\
\hline Suzuki et al ${ }^{38 *}$ & 2003 & Japan & Asian & PCR-RFLP & $\mathrm{HB}$ & $81 / 105$ & $Y$ & 6 \\
\hline Szendroi et al ${ }^{39}$ & 2011 & Hungary & Caucasian & PCR-RFLP & PB & $204 / 102$ & $Y$ & 7 \\
\hline Chen et $\mathrm{al}^{40 *}$ & 2009 & UK & Caucasian & TaqMan & $\mathrm{HB}$ & $\begin{array}{l}\text { Gleason score }<7 / \geq 7 \text { I I04/449 } \\
\text { Localized/Advanced I356//97 }\end{array}$ & Y & 7 \\
\hline Williams et al ${ }^{41 *}$ & 2004 & USA & Mixed & TaqMan & $\mathrm{HB}$ & $\begin{array}{l}\text { Gleason score }<7 / \geq 7 \quad 159 / 267 \\
\text { (Caucasian) and I02/208 } \\
\text { (African) }\end{array}$ & Y & 7 \\
\hline
\end{tabular}

Note: *These studies evaluated the association between Bsm I and PCa progression by different clinical stage or Gleason score. In the Sample size column the three numbers were case/ $\mathrm{HB} / \mathrm{BPH}$ as it has two control groups.

Abbreviations: HWE, Hardy-Weinberg equilibrium; NOS, Newcastle-Ottawa Scale; HB, hospital-based; BPH, benign prostate hyperplasia; PB, population-based; N, nonHWE; Y, HWE.

increased in patients with bb genotype or b genotype specifically in the subgroup of benign prostatic hyperplasia $(\mathrm{BPH})$ controls (Bb vs. bb: $\mathrm{OR}=0.689,95 \% \mathrm{CI}=0.534-0.890, p=0.004 ; \mathrm{BB} /$ $\mathrm{Bb}$ vs. bb: $\mathrm{OR}=0.627,95 \% \mathrm{CI}=0.411-0.954, p=0.029 ; \mathrm{B}$ vs. b: $\mathrm{OR}=0.715,95 \% \mathrm{CI}=0.530-0.965, p=0.029)$. However, the results for the other two control groups revealed no significant association (Table 5).

\section{Pooled results in terms of Bsm I polymorphism with $\mathrm{PCa}$ progression}

Stratified analyses, according to the clinical stages and Gleason score of patients, were also performed. As shown in Table 6, the pooled results for the patients with Gleason score $<7$ and Gleason score $\geq 7$ did not reveal any relationship between the
Bsm I variant and PCa risk in various genetic models compared to controls. Similarly, the subgroup of PCa cases with localized stage and aggressive stage showed no association.

In the inter-patient comparisons by different clinical stages and Gleason score statuses, a weak influence of Bsm I polymorphism on PCa progression was detected in patients with Gleason score $\geq 7$ compared to the group with Gleason score $<7$ (BB/Bb vs. bb: $\mathrm{OR}=1.176,95 \% \mathrm{CI}=1.008-1.373$, $p=0.04)$. However, no effect of Bsm I polymorphism on the clinical stages was detected (Figure 4 and Table 6).

\section{Publication bias and sensitivity analysis}

The funnel plots for publication bias analysis did not show any significant asymmetry in the overall analysis (Figure 5). 
Table 2 Results of the association between Bsm I polymorphism and PCa risk in the whole population

\begin{tabular}{|c|c|c|c|c|c|c|c|c|}
\hline \multirow[t]{2}{*}{ Comparison } & \multirow[t]{2}{*}{ Studies } & \multicolumn{3}{|l|}{ Overall effect } & \multicolumn{2}{|c|}{ Heterogeneity } & \multicolumn{2}{|c|}{ Publication bias } \\
\hline & & OR (95\% CI) & Z-score & $p$-value & $I^{2}(\%)$ & $p$-value & Begg's test & Egger's test \\
\hline BB vs. bb & 25 & $0.977(0.889-1.074)$ & 0.48 & 0.634 & 48.5 & 0.005 & 0.874 & 0.901 \\
\hline Bb vs. bb & 25 & $0.940(0.825-1.072)$ & 0.92 & 0.357 & 59.2 & 0 & 0.126 & 0.013 \\
\hline $\mathrm{BB} / \mathrm{Bb}$ vs. bb & 25 & 0.951 (0.832-I.087) & 0.74 & 0.462 & 65.9 & 0 & 0.229 & 0.042 \\
\hline $\mathrm{BB}$ vs. $\mathrm{Bb} / \mathrm{bb}$ & 25 & $1.002(0.923-1.087)$ & 0.05 & 0.963 & 12.3 & 0.293 & 0.853 & 0.824 \\
\hline B vs. b & 25 & $0.969(0.883-1.065)$ & 0.65 & 0.516 & 65.8 & 0 & 0.913 & 0.229 \\
\hline
\end{tabular}

Abbreviations: $\mathrm{PCa}$, prostate cancer; OR, odds ratio; $\mathrm{Cl}$, confidence interval.

Table 3 Results of the association between Bsm I polymorphism and PCa risk by different ethnicities

\begin{tabular}{|c|c|c|c|c|c|c|c|c|}
\hline \multirow[t]{2}{*}{ Comparison } & \multirow[t]{2}{*}{ Studies } & \multicolumn{3}{|l|}{ Overall effect } & \multicolumn{2}{|c|}{ Heterogeneity } & \multicolumn{2}{|c|}{ Publication bias } \\
\hline & & OR (95\% CI) & Z-score & $p$-value & $I^{2}(\%)$ & $p$-value & Begg's test & Egger's test \\
\hline \multicolumn{9}{|l|}{ Asian } \\
\hline BB vs. bb & II & $1.075(0.625-1.850)$ & 0.26 & 0.793 & 26.7 & 0.207 & 1 & 0.945 \\
\hline BB vs. bb & II & $0.884(0.592-I .320)$ & 0.6 & 0.546 & 65.9 & 0.001 & 0.484 & 0.253 \\
\hline $\mathrm{BB} / \mathrm{Bb}$ vs. bb & II & $0.913(0.612-1.362)$ & 0.45 & 0.656 & 69.9 & 0 & 0.392 & 0.371 \\
\hline$B B$ vs. $B b / b b$ & II & I.I25 (0.756-I.675) & 0.58 & 0.562 & 0.0 & 0.618 & 0.677 & 0.987 \\
\hline$B$ vs. $b$ & II & $0.957(0.686-1.334)$ & 0.26 & 0.794 & 70.0 & 0 & 0.938 & 0.481 \\
\hline \multicolumn{9}{|l|}{ Caucasian } \\
\hline BB vs. bb & II & $0.975(0.812-I .172)$ & 0.26 & 0.791 & 58.2 & 0.008 & 0.815 & 0.875 \\
\hline Bb vs. bb & II & $0.970(0.840-I .120)$ & 0.42 & 0.675 & 60.0 & 0.005 & 0.186 & 0.215 \\
\hline $\mathrm{BB} / \mathrm{Bb}$ vs. bb & II & $0.975(0.839-1.134)$ & 0.33 & 0.743 & 67.8 & 0.001 & 0.392 & 0.366 \\
\hline $\mathrm{BB}$ vs. $\mathrm{Bb} / \mathrm{bb}$ & II & $0.995(0.904-1.094)$ & 0.11 & 0.913 & 31.7 & 0.146 & 0.938 & 0.835 \\
\hline B vs. b & II & $0.98 \mid(0.887-I .085)$ & 0.37 & 0.711 & 67.2 & 0.001 & 0.938 & 0.649 \\
\hline \multicolumn{9}{|l|}{ African } \\
\hline BB vs. bb & 3 & I.I3I (0.316-4.055) & 0.19 & 0.85 & 83.1 & 0.003 & 0.117 & 0.137 \\
\hline Bb vs. bb & 3 & I.I3I (0.544-2.349) & 0.33 & 0.742 & 71.3 & 0.031 & 0.602 & 0.212 \\
\hline $\mathrm{BB} / \mathrm{Bb}$ vs. bb & 3 & I.I55 (0.509-2.622) & 0.34 & 0.731 & 79.7 & 0.007 & 0.602 & 0.273 \\
\hline $\mathrm{BB}$ vs. Bb/bb & 3 & I.02I (0.670-I.555) & 0.1 & 0.924 & 63.7 & 0.064 & 0.602 & 0.578 \\
\hline$B$ vs. $b$ & 3 & I.0I5 (0.592-I.738) & 0.05 & 0.957 & 80.6 & 0.006 & 0.117 & 0.228 \\
\hline
\end{tabular}

Abbreviations: $\mathrm{PCa}$, prostate cancer; OR, odds ratio; $\mathrm{Cl}$, confidence interval.

Table 4 Results of the association between Bsm I polymorphism and PCa risk by different genotyping methods

\begin{tabular}{|c|c|c|c|c|c|c|c|c|}
\hline \multirow[t]{2}{*}{ Comparison } & \multirow[t]{2}{*}{ Studies } & \multicolumn{3}{|l|}{ Overall effect } & \multicolumn{2}{|c|}{ Heterogeneity } & \multicolumn{2}{|c|}{ Publication bias } \\
\hline & & OR (95\% CI) & Z-score & $p$-value & $P^{2}(\%)$ & $p$-value & Begg's test & Egger's test \\
\hline \multicolumn{9}{|l|}{ PCR-RFLP } \\
\hline BB vs. bb & 16 & $0.979(0.730-1.313)$ & 0.14 & 0.877 & 60.7 & 0.001 & 0.528 & 0.742 \\
\hline BB vs. bb & 16 & $0.976(0.784-1.215)$ & 0.22 & 0.826 & 68.1 & 0 & 0.528 & 0.758 \\
\hline $\mathrm{BB} / \mathrm{Bb}$ vs. bb & 16 & $0.987(0.789-1.235)$ & 0.11 & 0.91 & 73.7 & 0 & 0.510 & 0.513 \\
\hline $\mathrm{BB}$ vs. $\mathrm{Bb} / \mathrm{bb}$ & 16 & $0.987(0.861-1.132)$ & 0.19 & 0.853 & 35.7 & 0.077 & 0.510 & 0.513 \\
\hline B vs. b & 16 & $0.995(0.842-1.176)$ & 0.06 & 0.951 & 74.4 & 0 & 0.510 & 0.569 \\
\hline \multicolumn{9}{|l|}{ TaqMan } \\
\hline BB vs. bb & 3 & $1.04 \mid(0.867-1.250)$ & 0.43 & 0.668 & 0.0 & 0.71 & 0.117 & 0.126 \\
\hline Bb vs. bb & 3 & $0.957(0.838-1.093)$ & 0.65 & 0.518 & 0.0 & 0.948 & 0.117 & 0.016 \\
\hline $\mathrm{BB} / \mathrm{Bb}$ vs. bb & 3 & $0.972(0.859-1.100)$ & 0.45 & 0.656 & 0.0 & 0.84 & 0.117 & 0.48 \\
\hline $\mathrm{BB}$ vs. $\mathrm{Bb} / \mathrm{bb}$ & 3 & 1.031 (0.893-1.191) & 0.42 & 0.677 & 0.0 & 0.689 & 0.117 & 0.48 \\
\hline B vs. b & 3 & $0.998(0.918-1.084)$ & 0.06 & 0.954 & 0.0 & 0.696 & 0.117 & 0.316 \\
\hline \multicolumn{9}{|l|}{ SNPlex } \\
\hline BB vs. bb & 4 & $0.960(0.786-1 .|7|)$ & 0.41 & 0.685 & 13.1 & 0.327 & 0.497 & 0.501 \\
\hline $\mathrm{Bb}$ vs. bb & 4 & $0.852(0.67 \mid-1.082)$ & 1.31 & 0.19 & 54.9 & 0.064 & 0.497 & 0.492 \\
\hline $\mathrm{BB} / \mathrm{Bb}$ vs. bb & 4 & $0.854(0.677-1.078)$ & 1.33 & 0.184 & 57.0 & 0.054 & 0.624 & 0.427 \\
\hline $\mathrm{BB}$ vs. $\mathrm{Bb} / \mathrm{bb}$ & 4 & $0.990(0.857-1.143)$ & 0.14 & 0.888 & 0.0 & 0.861 & 0.624 & 0.513 \\
\hline B vs. b & 4 & $0.918(0.796-1.060)$ & 1.17 & 0.243 & 50.5 & 0.089 & 0.070 & 0.126 \\
\hline
\end{tabular}

Abbreviations: $\mathrm{PCa}$, prostate cancer; OR, odds ratio; $\mathrm{Cl}$, confidence interval. 
Table 5 Results of the association between Bsm I polymorphism and PCa risk by different sources of controls

\begin{tabular}{|c|c|c|c|c|c|c|c|c|}
\hline \multirow[t]{2}{*}{ Comparison } & \multirow[t]{2}{*}{ Studies } & \multicolumn{3}{|l|}{ Overall effect } & \multicolumn{2}{|c|}{ Heterogeneity } & \multicolumn{2}{|c|}{ Publication bias } \\
\hline & & OR (95\% CI) & Z-score & $p$-value & $I^{2}(\%)$ & $p$-value & Begg's test & Egger's test \\
\hline \multicolumn{9}{|c|}{ Population-based } \\
\hline BB vs. bb & 17 & $0.963(0.809-1.147)$ & 0.42 & 0.675 & 48.5 & 0.016 & 0.653 & 0.737 \\
\hline Bb vs. bb & 17 & $0.910(0.779-1.065)$ & 1.18 & 0.24 & 69.5 & 0 & 0.510 & 0.599 \\
\hline $\mathrm{BB} / \mathrm{Bb}$ vs. bb & 17 & $0.920(0.787-1.075)$ & 1.05 & 0.294 & 73.0 & 0 & 0.742 & 0.656 \\
\hline $\mathrm{BB}$ vs. $\mathrm{Bb} / \mathrm{bb}$ & 17 & I.004 (0.909-I.109) & 0.08 & 0.937 & 14.0 & 0.293 & 0.928 & 0.961 \\
\hline$B$ vs. $b$ & 17 & $0.950(0.850-1.062)$ & 0.9 & 0.368 & 71.9 & 0 & I & 0.968 \\
\hline \multicolumn{9}{|c|}{ Hospital-based } \\
\hline BB vs. bb & 6 & $0.95 I(0.505-1.790)$ & 0.15 & 0.877 & 43.1 & 0.118 & 0.573 & 0.973 \\
\hline BB vs. bb & 6 & $1.016(0.600-1.721)$ & 0.06 & 0.953 & 70.6 & 0.005 & 0.573 & 0.782 \\
\hline $\mathrm{BB} / \mathrm{Bb}$ vs. bb & 6 & $\mathrm{I} .026(0.607-\mathrm{I} .732)$ & 0.1 & 0.924 & 75.0 & 0.001 & 0.851 & 0.858 \\
\hline $\mathrm{BB}$ vs. $\mathrm{Bb} / \mathrm{bb}$ & 6 & $1.015(0.806-1.279)$ & 0.13 & 0.879 & 30.9 & 0.204 & 0.573 & 0.969 \\
\hline$B$ vs. $b$ & 6 & $1.035(0.666-1.607)$ & 0.15 & 0.879 & 78.1 & 0 & 0.851 & 0.906 \\
\hline \multicolumn{9}{|l|}{$\mathbf{B P H}$} \\
\hline BB vs. bb & 6 & $0.515(0.239-1.108)$ & 1.7 & 0.09 & 67.7 & 0.015 & 0.624 & 0.287 \\
\hline Bb vs. bb & 6 & $0.689(0.534-0.890)$ & 2.86 & 0.004 & 42.8 & 0.12 & 0.573 & 0.325 \\
\hline $\mathrm{BB} / \mathrm{Bb}$ vs. bb & 6 & $0.627(0.4 I \mid-0.954)$ & 2.18 & 0.029 & 56.7 & 0.042 & 0.573 & 0.27 \\
\hline $\mathrm{BB}$ vs. $\mathrm{Bb} / \mathrm{bb}$ & 6 & $0.842(0.649-1.093)$ & 1.29 & 0.197 & 28.6 & 0.231 & I & 0.385 \\
\hline B vs. b & 6 & $0.7 \mid 5(0.530-0.965)$ & 2.19 & 0.029 & 59.1 & 0.032 & 0.573 & 0.379 \\
\hline
\end{tabular}

Note: Bold values indicate statistical significance.

Abbreviations: PCa, prostate cancer; OR, odds ratio; Cl, confidence interval; BPH, benign prostatic hyperplasia.

Moreover, Begg's and Egger's tests also revealed no publication bias in overall analysis as well as subgroup analyses (Tables 2-6). Sensitivity analysis for the positive results suggested that no obvious change in the pooled results was detected by omitting each individual study for the subgroup analysis of BPH controls, while the results were unstable in the comparison of PCa cases in terms of Gleason scores (Figure 6).

\section{Discussion}

Polymorphisms of $V D R$ gene and their relationships with $\mathrm{PCa}$ susceptibility have drawn a lot of attention in recent years. Bsm I polymorphism is one of the "star biomarkers". Even though Bsm I polymorphism is located in the noncoding regions of $V D R$ gene, it is frequently considered to be associated with PCa risk by numerous studies..$^{9,11,12,39}$ Meanwhile, some studies support the opposite conclusion. ${ }^{15,16,30,34}$ Five meta-analyses conducted by Yin et al, ${ }^{19}$ Zhang et al, ${ }^{20}$ Guo et al, ${ }^{21} \mathrm{Xu}$ et al, ${ }^{22}$ and Liu et al, ${ }^{10}$ including $14,19,19,15$, and 6 primary studies, respectively, also yielded conflicting results. Moreover, some new data were reported. ${ }^{3,9,14,23,24}$ Therefore, a new meta-analysis is necessary to clarify this issue. In the present study, data of 27 independent studies including 9,993 cases and 9,345 controls, which is higher compared to the previous meta-analyses, were pooled. Therefore, our updated results will be more convincing and stringent.

According to our results, no association between $\mathrm{PCa}$ risk and Bsm I polymorphism was detected in the overall population, which was similar to the results reported by Guo et al, ${ }^{21}$ Liu et al,${ }^{10}$ and Xu et al, ${ }^{22}$ but different from the other two meta-analyses. ${ }^{19,20}$ As we mentioned above, the results of previous meta-analyses might be suspect due to outdated data or inclusion of incomplete studies. Ethnicity might be an important biological factor for the genetic difference. ${ }^{42}$ The genotype frequency distribution of $B s m I$ was found to be different between Asians, Caucasians and Africans, but in each subgroup by ethnicity, no association was found. In addition, subgroup analyses by the genotyping method and HWE status both revealed no influence of Bsm I on PCa risk, suggesting that these two variables would not change the negative result of the overall analysis either.

An interesting finding was that according to the results of the subgroup analysis by different sources of controls, Bsm I mutation increased the risk of PCa in BPH controls in the heterozygote model, recessive model, and allele model. Moreover, this result was proved to be robust by sensitivity analysis, and the heterogeneity was found to be acceptable as well. Based on this result, for individuals with BPH, the bb genotype or $\mathrm{b}$ might increase the risk of $\mathrm{PCa}$, however, this result was suspicious and difficult to explain. Age was reported to be a risk factor for the relationship between $B s m$ $I$ mutation and $\mathrm{PCa}$ risk. ${ }^{26,35} \mathrm{We}$ intended to perform a subgroup meta-analysis by age, but the age classification in the included studies was too ambiguous to be pooled.

Similar to overall analysis, subgroup analyses by clinical stage and Gleason score revealed no relationship between 
Table 6 Results of the association between Bsm I polymorphism and PCa risk by different tumor stages

\begin{tabular}{|c|c|c|c|c|c|c|c|c|}
\hline \multirow[t]{2}{*}{ Comparison } & \multirow[t]{2}{*}{ Studies } & \multicolumn{3}{|l|}{ Overall effect } & \multicolumn{2}{|c|}{ Heterogeneity } & \multicolumn{2}{|c|}{ Publication bias } \\
\hline & & OR (95\% Cl) & Z-score & $\overline{p \text {-value }}$ & $I^{2}(\%)$ & $p$-value & Begg's test & Egger's test \\
\hline \multicolumn{9}{|c|}{ Gleason score $<7$ (cases vs. controls) } \\
\hline BB vs. bb & 6 & $1.095(0.490-2.449)$ & 0.22 & 0.824 & 74.8 & 0.001 & $0.85 I$ & 0.4 \\
\hline Bb vs. bb & 6 & $1.05 \mid(0.848-1.304)$ & 0.46 & 0.649 & 28.6 & 0.22 & 0.573 & 0.072 \\
\hline $\mathrm{BB} / \mathrm{Bb}$ vs. bb & 6 & $0.942(0.612-1.450)$ & 0.27 & 0.787 & 69.4 & 0.006 & 0.348 & 0.147 \\
\hline $\mathrm{BB}$ vs. $\mathrm{Bb} / \mathrm{bb}$ & 6 & $1.097(0.572-2.106)$ & 0.28 & 0.78 & 66.8 & 0.01 & $0.85 I$ & 0.426 \\
\hline B vs. b & 6 & $0.957(0.635-1.443)$ & 0.21 & 0.835 & 81.1 & 0 & 0.573 & 0.193 \\
\hline \multicolumn{9}{|c|}{ Gleason score $\geq 7$ (cases vs. controls) } \\
\hline BB vs. bb & 6 & $0.873(0.607-1.253)$ & 0.74 & 0.46 & 0.0 & 0.429 & 0.573 & 0.899 \\
\hline Bb vs. bb & 6 & $0.787(0.609-1.017)$ & 1.83 & 0.067 & 55.3 & 0.048 & 0.348 & 0.15 \\
\hline $\mathrm{BB} / \mathrm{Bb}$ vs. bb & 6 & $0.742(0.470-\mid .17 I)$ & 1.28 & 0.2 & 63.1 & 0.019 & 0.039 & 0.191 \\
\hline $\mathrm{BB}$ vs. $\mathrm{Bb} / \mathrm{bb}$ & 6 & $0.920(0.662-1.279)$ & 0.48 & 0.621 & 0.0 & 0.483 & 0.851 & 0.874 \\
\hline B vs. b & 6 & $0.793(0.540-1.163)$ & 1.19 & 0.235 & 69.2 & 0.006 & 0.091 & 0.253 \\
\hline \multicolumn{9}{|c|}{ Localized (cases vs. controls) } \\
\hline BB vs. bb & 6 & $0.855(0.632-1.158)$ & 1.01 & 0.312 & 47.9 & 0.088 & 0.851 & 0.478 \\
\hline $\mathrm{Bb}$ vs. bb & 6 & $0.793(0.627-1.003)$ & 1.93 & 0.053 & 0.0 & 0.78 & 0.188 & 0.19 \\
\hline $\mathrm{BB} / \mathrm{Bb}$ vs. bb & 6 & $0.818(0.661-1.012)$ & 1.85 & 0.065 & 0.0 & 0.504 & 0.348 & 0.216 \\
\hline $\mathrm{BB}$ vs. $\mathrm{Bb} / \mathrm{bb}$ & 6 & $0.923(0.701-1.215)$ & 0.57 & 0.567 & 45.7 & 0.101 & 0.851 & 0.443 \\
\hline B vs. b & 6 & $0.874(0.748-1.022)$ & 1.69 & 0.092 & 42.8 & 0.12 & 0.188 & 0.22 \\
\hline \multicolumn{9}{|c|}{ Aggressive (cases vs. controls) } \\
\hline BB vs. bb & 6 & $0.709(0.46 \mathrm{I}-\mathrm{I} .092)$ & 1.56 & 0.118 & 0.0 & 0.637 & 0.573 & 0.339 \\
\hline $\mathrm{Bb}$ vs. bb & 6 & $0.753(0.559-1.014)$ & 1.87 & 0.062 & 54.7 & 0.051 & 0.348 & 0.466 \\
\hline $\mathrm{BB} / \mathrm{Bb}$ vs. bb & 6 & $0.693(0.416-1.155)$ & 1.41 & 0.159 & 54.9 & 0.05 & 0.851 & 0.799 \\
\hline $\mathrm{BB}$ vs. $\mathrm{Bb} / \mathrm{bb}$ & 6 & $0.785(0.530-1.164)$ & 1.20 & 0.229 & 0.0 & 0.685 & 0.851 & 0.323 \\
\hline B vs. b & 6 & $0.711(0.459-1.101)$ & 1.53 & 0.127 & 56.7 & 0.042 & 0.573 & 0.603 \\
\hline \multicolumn{9}{|c|}{ Gleason score $\geq 7$ vs. $<7$} \\
\hline BB vs. bb & 8 & $1.207(0.962-1.5 \mid 4)$ & 1.63 & 0.103 & 53.8 & 0.043 & 0.548 & 0.632 \\
\hline Bb vs. bb & 8 & I.I 66 (0.989-I.375) & 1.82 & 0.068 & 18.0 & 0.288 & 0.266 & 0.684 \\
\hline $\mathrm{BB} / \mathrm{Bb}$ vs. bb & 8 & $1.176(1.008-1.373)$ & 2.06 & 0.040 & 39.3 & 0.117 & 0.536 & 0.763 \\
\hline $\mathrm{BB}$ vs. $\mathrm{Bb} / \mathrm{bb}$ & 8 & 1.131 (0.919-1.392) & 1.16 & 0.246 & 51.1 & 0.056 & $\mathrm{I}$ & 0.833 \\
\hline B vs. b & 8 & $1.163(0.928-1.457)$ & 1.31 & 0.191 & 59.9 & 0.015 & 0.536 & 0.901 \\
\hline \multicolumn{9}{|c|}{ Aggressive vs. localized } \\
\hline BB vs. bb & 7 & $0.946(0.692-1.295)$ & 0.34 & 0.731 & 22.0 & 0.268 & 0.133 & 0.054 \\
\hline Bb vs. bb & 7 & $0.97 \mid(0.765-\mid .23 I)$ & 0.806 & 0.25 & 23.0 & 0.254 & 0.368 & 0.338 \\
\hline $\mathrm{BB} / \mathrm{Bb}$ vs. bb & 7 & $0.984(0.790-1.226)$ & 0.14 & 0.887 & 4.0 & 0.396 & 0.035 & 0.031 \\
\hline $\mathrm{BB}$ vs. $\mathrm{Bb} / \mathrm{bb}$ & 7 & $0.966(0.727-1.284)$ & 0.24 & 0.812 & 23.7 & 0.256 & 0.133 & 0.071 \\
\hline B vs. b & 7 & $0.98 \mid(0.839-1.147)$ & 0.24 & 0.808 & 26.9 & 0.224 & 0.035 & 0.001 \\
\hline
\end{tabular}

Note: Bold values indicate statistical significance.

Abbreviations: $\mathrm{PCa}$, prostate cancer; OR, odds ratio; $\mathrm{Cl}$, confidence interval.

PCa risk and Bsm I. Moreover, we conducted inter-patient analysis to assess the relationship of Bsm I polymorphism with PCa progression by comparing cases with aggressive stage and Gleason score $\geq 7$ to cases with localized stage and Gleason score $<7$, respectively. Almost all the results were negative, except for the comparison between cases with Gleason score $\geq 7$ and $<7$ in the recessive model. However, the only positive result was not stable in sensitivity analysis. Therefore, we have ignored the weak relationship.

Regrettably, we failed to perform a subgroup analysis by vitamin D intake, because only two primary studies have a detailed description of the effect of plasma vitamin $\mathrm{D}$ levels on the association between Bsm I and PCa risk. Ma et al reported that in patients with low levels of $25-\mathrm{D}$, which is one of the vitamin D metabolites, the PCa risk would be significantly increased by carrying bb genotype. ${ }^{33}$ Meanwhile, in the group with high levels of 25-D, the relationship was not significant. Similar results were reported by Ahn et al in 2009. ${ }^{18}$ These studies suggest that plasma levels of 25-D might influence our pooled result, and a stratified analysis by vitamin D intake or 25-D levels is warranted in the future.

Significant heterogeneity between studies was detected in both overall analysis and subgroup analyses under multiple comparison models. We noted that the BB genotype in the Asian group was quite rare but very commonly detected in Caucasians and Africans. It may contribute to this heterogeneity. 
A

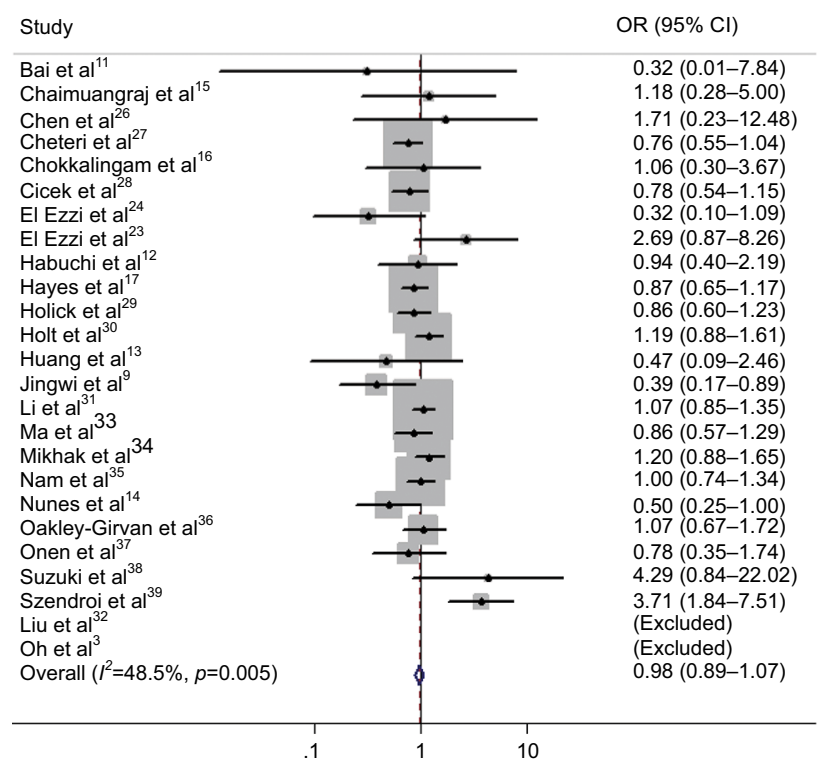

B

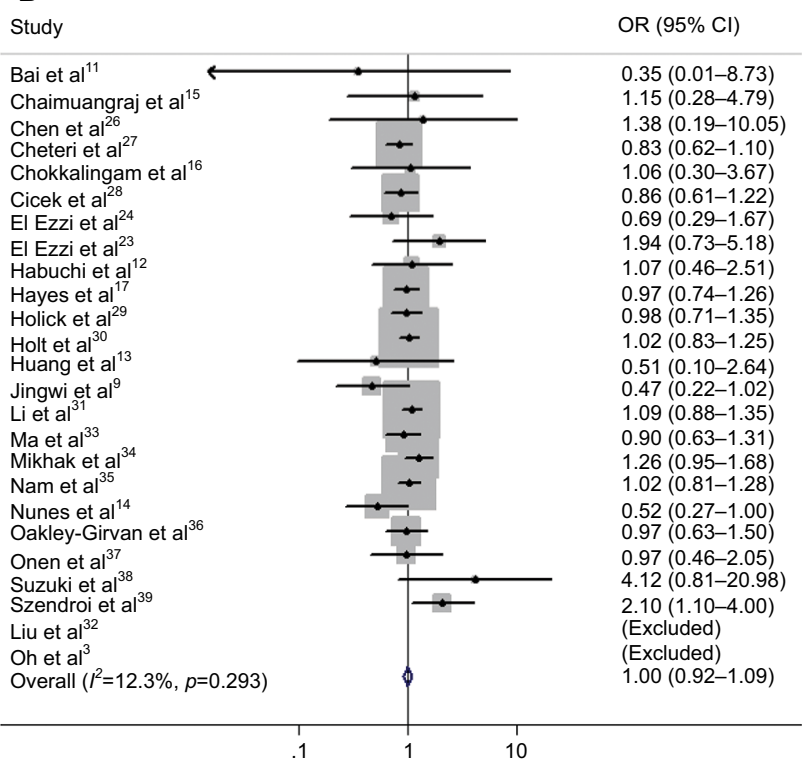

Figure 2 Forest plots to estimate the association of VDR Bsm I polymorphism with PCa in the overall analysis. (A) Homozygote model (BB vs. bb). (B) Recessive model (BB vs. Bb/bb).

Abbreviations: PCa, prostate cancer; OR, odds ratio; $\mathrm{Cl}$, confidence interval.

A Study

\begin{tabular}{|c|c|}
\hline Chaimuangraj et al ${ }^{15}$ & $1.33(0.32-5.53)$ \\
\hline El Ezzi et al ${ }^{24}$ & $0.38(0.13-1.08)$ \\
\hline Habuchi et al ${ }^{12}$ & $0.66(0.41-1.04)$ \\
\hline Nam et al ${ }^{35}$ & $0.94(0.62-1.40)$ \\
\hline Nunes et al ${ }^{14}$ & $0.23(0.08-0.64)$ \\
\hline Oh et $\mathrm{al}^{3}$ & $0.66(0.31-1.41)$ \\
\hline Overall $\left(I^{2}=42.8 \%, p=0.120\right)$ & $0.69(0.53-0.89)$ \\
\hline
\end{tabular}

B

Study

OR $(95 \% \mathrm{Cl})$

\begin{tabular}{|c|c|}
\hline Chaimuangraj et al ${ }^{15}$ & $1.25(0.51-3.09)$ \\
\hline El Ezzi et al ${ }^{24}$ & $0.67(0.40-1.129)$ \\
\hline Habuchi et al ${ }^{12}$ & $0.70(0.48-1.01)$ \\
\hline Nam et al ${ }^{35}$ & $0.95(0.77-1.18)$ \\
\hline Nunes et $\mathrm{al}^{14}$ & $0.39(0.24-0.65)$ \\
\hline Oh et $\mathrm{al}^{3}$ & $0.67(0.32-1.41)$ \\
\hline Overall $\left(I^{2}=59.1 \%, p=0\right.$ & $0.72(0.53-0.97)$ \\
\hline
\end{tabular}

Figure 3 Forest plots to estimate the association of VDR Bsm I polymorphism with PCa in the subgroup of BPH controls. (A) Heterozygote model (Bb vs. bb). (B) Allelic frequency model (B vs. b).

Abbreviations: $\mathrm{PCa}$, prostate cancer; $\mathrm{BPH}$, benign prostatic hyperplasia; $\mathrm{OR}$, odds ratio; $\mathrm{Cl}$, confidence interval.

However, no obvious publication bias was found and the sensitivity analysis supported the stability of our results. Overall, the present analysis was credible and statistically valid for the studied population.

However, some limitations of our meta-analysis should be acknowledged. First of all, some reports with a small number of cases and controls were included in our analysis, which increases the statistical power but introduces potential bias and heterogeneity as well. Second, our pooled outcomes were based on the initial results of the included studies, which were not adjusted by patient characteristics and other potential factors, such as age, gender, smoking, alcohol, sunshine, vitamin D intake, and so on. Therefore, a more precise analysis is required, in which the results should be adjusted by some related parameters. Besides, heterogeneity was obviously detected in some pooled results, which cannot be eliminated by subgroup analyses.

In conclusion, the present pooled analysis might be the largest one so far to evaluate the relationship between PCa susceptibility and Bsm I polymorphism of $V D R$ gene. No increased risk of PCa was detected to be associated with Bsm I mutant in the overall analysis, and similarly in different 
Study

\begin{tabular}{|c|c|}
\hline Bai et al ${ }^{11}$ & $3.33(0.76-14.69)$ \\
\hline Cheteri et $\mathrm{al}^{27}$ & $1.93(1.15-3.24)$ \\
\hline Cicek et $\mathrm{al}^{28}$ & $0.87(0.59-1.30)$ \\
\hline Huang et $\mathrm{al}^{13}$ & $2.87(0.76-10.85)$ \\
\hline Nunes et $\mathrm{al}^{14}$ & $1.03(0.47-2.22)$ \\
\hline Suzuki 2003 & $0.62(0.20-1.92)$ \\
\hline Chen 2009 & $1.24(0.99-1.56)$ \\
\hline Williams et al ${ }^{41}$ & $1.03(0.73-1.44)$ \\
\hline Overall $\left(I^{2}=39.3 \%, p=0.117\right)$ & $1.18(1.01-1.37)$ \\
\hline
\end{tabular}

Figure 4 Forest plot to estimate the association of VDR Bsm I polymorphism with cases with Gleason score $>7$ and cases with Gleason score $<7$ in the dominant model (BB/Bb vs. bb).

Abbreviations: $\mathrm{OR}$, odds ratio; $\mathrm{Cl}$, confidence interval.

A Begg's funnel plot with pseudo $95 \%$ confidence limits

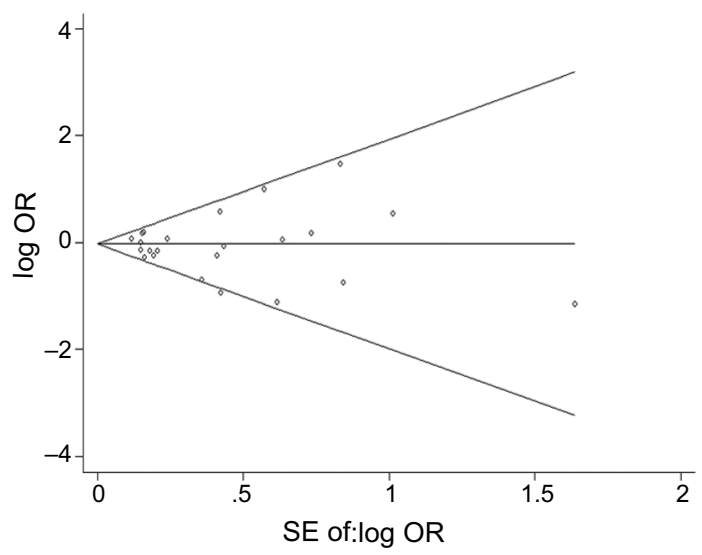

B Begg's funnel plot with pseudo $95 \%$ confidence limits

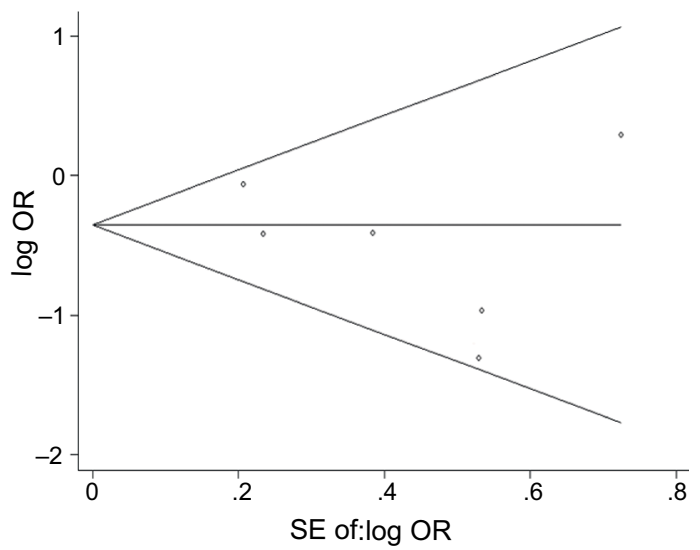

Figure 5 Begg's funnel plots to examine publication bias for reported comparisons of VDR gene Bsm I polymorphism for the homozygote model in the (A) overall analysis and $(\mathbf{B})$ the subgroup analysis of BPH controls.

Abbreviations: $\mathrm{BPH}$, benign prostatic hyperplasia; OR, odds ratio.
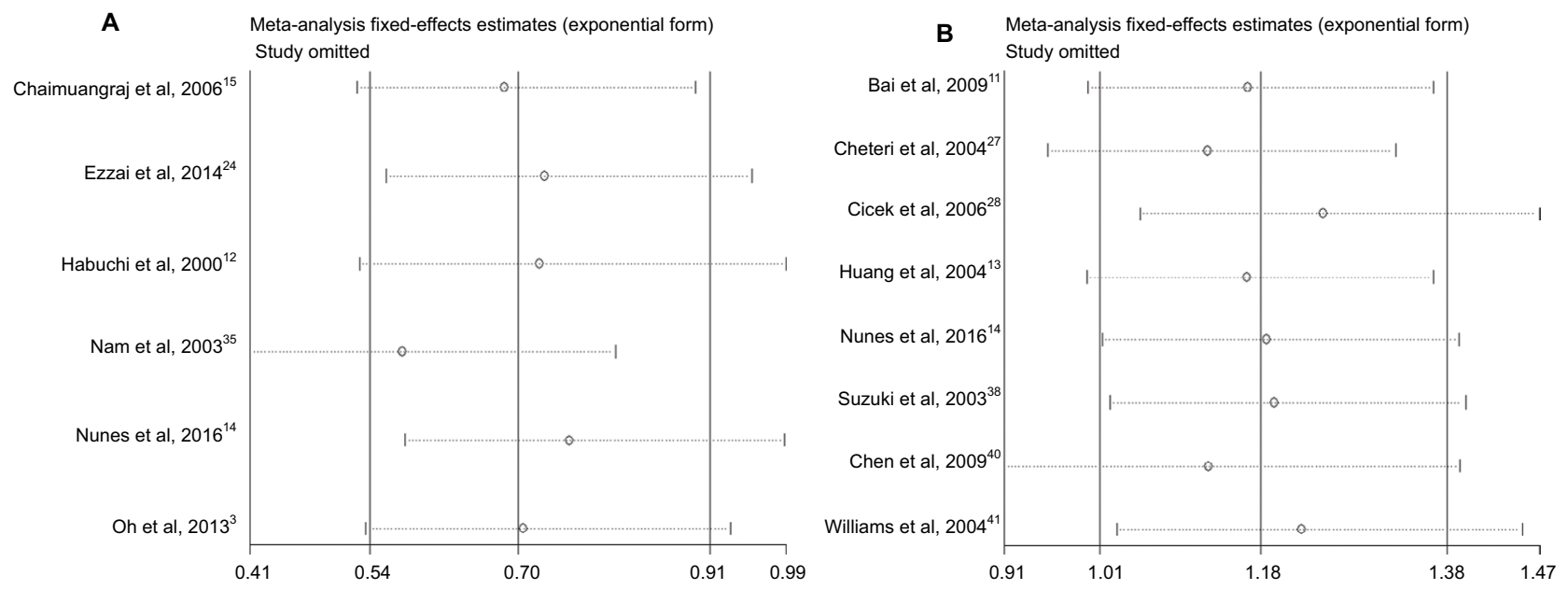

Figure 6 Sensitivity analysis of the (A) comparison of $\mathrm{PCa}$ cases with $\mathrm{BPH}$ controls (Bb vs. bb) and (B) comparison of $\mathrm{PCa}$ cases with $\mathrm{Gleason}$ score $>7$ vs. $<7$ (BB/Bb vs. bb) Abbreviations: $\mathrm{PCa}$, prostate cancer; $\mathrm{BPH}$, benign prostatic hyperplasia. 
subgroup analyses by race, genotyping methods, HWE status of controls, and clinical stage and Gleason score of cases. The association between PCa progression and Bsm I was also negative. Individuals with $\mathrm{BPH}$, carrying bb genotype and $b$, seemed to have an increased risk of PCa. More large-scale and well-designed studies are needed in future to demonstrate the weak influence of Bsm I mutant on PCa risk and progression.

\section{Author contributions}

All authors contributed toward data analysis, drafting and revising the paper and agree to be accountable for all aspects of the work.

\section{Disclosure}

The authors report no conflicts of interest in this work.

\section{References}

1. Siegel RL, Miller KD, Jemal A. Cancer statistics. CA Cancer J Clin. 2018;68(1):7-30.

2. Wang $\mathrm{K}, \mathrm{Wu} \mathrm{G}, \mathrm{Li}$ J, et al. Role of vitamin D receptor gene Cdx2 and Apa1 polymorphisms in prostate cancer susceptibility: a meta-analysis. BMC Cancer. 2016;16(1):674.

3. Oh JJ, Byun SS, Lee SE, et al. Genetic variations in VDR associated with prostate cancer risk and progression in a Korean population. Gene. 2014;533(1):86-93.

4. Galunska B, Gerova D, Kosev P, et al. Serum 25-hydroxy vitamin D levels in Bulgarian patients with prostate cancer: a pilot study. Clin Lab. 2015;61(3-4):329-335.

5. Brandstedt J, Almquist M, Manjer J, et al. Vitamin D, PTH, and calcium in relation to survival following prostate cancer. Cancer Causes Control. 2016;27(5):669-777.

6. Chakraborti CK. Vitamin D as a promising anticancer agent. Indian J Pharmacol. 2011;43(2):113-120.

7. Kang S, Zhao Y, Liu J, et al. Association of vitamin D receptor Fok I polymorphism with the risk of prostate cancer: a meta-analysis. Oncotarget. 2016;7(47):77878-77889.

8. Tayeb MT, Clark C, Haites NE, et al. Vitamin D receptor, HER-2 polymorphisms and risk of prostate cancer in men with benign prostate hyperplasia. Saudi Med J. 2004;25(4):447-451.

9. Jingwi EY, Abbas M, Ricks-Santi L, et al. Vitamin D receptor genetic polymorphisms are associated with PSA level, Gleason score and prostate cancer risk in African-American men. Anticancer Res. 2015;35(3):1549-1558.

10. Liu S, Cai H, Cheng W, et al. Association of VDR polymorphisms (Taq I and Bsm I) with prostate cancer: a new meta-analysis. J Int Med Res. 2017;45(1):3-10.

11. Bai Y, Yu Y, Yu B, et al. Association of vitamin D receptor polymorphisms with the risk of prostate cancer in the Han population of Southern China. BMC Med Genet. 2009;10:125.

12. Habuchi T, Suzuki T, Sasaki R, et al. Association of vitamin D receptor gene polymorphism with prostate cancer and benign prostatic hyperplasia in a Japanese population. Cancer Res. 2000;60(2): 305-308.

13. Huang SP, Chou YH, Wayne Chang WS, et al. Association between vitamin D receptor polymorphisms and prostate cancer risk in a Taiwanese population. Cancer Lett. 2004;207(1):69-77.
14. Nunes SB, de Matos Oliveira F, Neves AF, et al. Association of vitamin $\mathrm{D}$ receptor variants with clinical parameters in prostate cancer. Springerplus. 2016;53:64

15. Chaimuangraj $\mathrm{S}$, Thammachoti $\mathrm{R}$, Ongphiphadhanakul $\mathrm{B}$, et al. Lack of association of $V D R$ polymorphisms with Thai prostate cancer as compared with benign prostate hyperplasia and controls. Asian Pac J Cancer Prev. 2006;7(1):136-139.

16. Chokkalingam AP, McGlynn KA, Gao YT, et al. Vitamin D receptor gene polymorphisms, insulin-like growth factors, and prostate cancer risk: a population-based case-control study in China. Cancer Res. 2001;61(11):4333-4336.

17. Hayes VM, Severi G, Padilla EJ, et al. Genetic variants in the vitamin D receptor gene and prostate cancer risk. Cancer Epidemiol Biomarkers Prev. 2005:14(4):997-999.

18. Ahn J, Albanes D, Berndt SI, et al. Vitamin D-related genes, serum vitamin D concentrations and prostate cancer risk. Carcinogenesis. 2009;30(5):769-776.

19. Yin M, Wei S, Wei Q. Vitamin D receptor genetic polymorphisms and prostate cancer risk: a meta-analysis of 36 published studies. Int J Clin Exp Med. 2009;2(2):159-175.

20. Zhang Q, Shan Y. Genetic polymorphisms of vitamin D receptor and the risk of prostate cancer: a meta-analysis. J BUON. 2013;18(4): 961-969.

21. Guo Z, Wen J, Kan Q, et al. Lack of association between vitamin D receptor gene Fok I and Bsm I polymorphisms and prostate cancer risk: an updated meta-analysis involving 21,756 subjects. Tumour Biol. 2013;34(5):3189-3200.

22. Xu Y, He B, Pan Y, et al. Systematic review and meta-analysis on vitamin D receptor polymorphisms and cancer risk. Tumour Biol. 2014;35(5):4153-4169.

23. El Ezzi AA, Baker MT, Zaidan WR, et al. Association of polymorphisms in the $V D R, C Y P 17$ and SRD5A2 genes and prostate cancer among Lebanese men. Asian Pac J Cancer Prev. 2017;18(1):93-100.

24. El Ezzi AA, Zaidan WR, El-Saidi MA, et al. Association of benign prostate hyperplasia with polymorphisms in VDR, CYP17, and SRD5A2 genes among Lebanese men. Asian Pac J Cancer Prev. 2014;15(3):1255-1262.

25. Bohning D. Meta-analysis: a unifying meta-likelihood approach framing unobserved heterogeneity, study covariates, publication bias, and study quality. Methods Inf Med. 2005;44(1):127-135.

26. Chen ZQ, Deng LS, Lin WJ. The association of vitamin D receptor genotypes and risk of prostate cancer. Chin J Lab Med Clin Sci. 2001;2(2):60-62.

27. Cheteri MB, Stanford JL, Friedrichsen DM, et al. Vitamin D receptor gene polymorphisms and prostate cancer risk. Prostate. 2004;59(4):409-418.

28. Cicek MS, Liu X, Schumacher FR, et al. Vitamin D receptor genotypes/ haplotypes and prostate cancer risk. Cancer Epidemiol Biomarkers Prev. 2006;15(12):2549-2552.

29. Holick CN, Stanford JL, Kwon EM, et al. Comprehensive association analysis of the vitamin D pathway genes, $V D R, C Y P 27 B 1$, and CYP24A1, in prostate cancer. Cancer Epidemiol Biomarkers Prev. 2007;16(10):1990-1999.

30. Holt SK, Kwon EM, Peters U, et al. Vitamin D pathway gene variants and prostate cancer risk. Cancer Epidemiol Biomarkers Prev. 2009;18(6):1929-1933.

31. Li H, Stampfer MJ, Hollis JB, et al. A prospective study of plasma vitamin D metabolites, vitamin D receptor polymorphisms, and prostate cancer. PLoS Med. 2007;4(3):e103.

32. Liu JH, Li WH, Wang QJ, et al. Vitamin D receptor gene Bsm I polymorphism and the suscepbility to prostate cancer in Northern Chinese Han population. Natl J Androl. 2003;9(6):413-416.

33. Ma J, Stampfer MJ, Gann PH, et al. Vitamin D receptor polymorphisms, circulating vitamin D metabolites, and risk of prostate cancer in United States physicians. Cancer Epidemiol Biomarkers Prev. 1998;7(5):385-390. 
34. Mikhak B, Hunter DJ, Spiegelman D, et al. Vitamin D receptor $(V D R)$ gene polymorphisms and haplotypes, interactions with plasma 25-hydroxyvitamin $\mathrm{D}$ and 1,25-dihydroxyvitamin $\mathrm{D}$, and prostate cancer risk. Prostate. 2007;67(9):911-923.

35. Nam RK, Zhang WW, Trachtenberg J, et al. Comprehensive assessment of candidate genes and serological markers for the detection of prostate cancer. Cancer Epidemiol Biomarkers Prev. 2003;12(12):1429-1437.

36. Oakley-Girvan I, Feldman D, Eccleshall TR, et al. Risk of early-onset prostate cancer in relation to germ line polymorphisms of the vitamin D receptor. Cancer Epidemiol Biomarkers Prev. 2004;13(8):1325-1330.

37. Onen IH, Ekmekci A, Eroglu M, et al. Association of genetic polymorphisms in vitamin D receptor gene and susceptibility to sporadic prostate cancer. Exp Biol Med (Maywood). 2008;233(12):1608-1614.

38. Suzuki K, Matsui H, Ohtake N, et al. Vitamin D receptor gene polymorphism in familial prostate cancer in a Japanese population. Int $J$ Urol. 2003;10(5):261-266.
39. Szendroi A, Speer G, Tabak A, et al. The role of vitamin D, estrogen, calcium sensing receptor genotypes and serum calcium in the pathogenesis of prostate cancer. Can J Urol. 2011;18(3):5710-5716.

40. Chen L, Davey Smith G, Evans DM, et al. Genetic variants in the vitamin d receptor are associated with advanced prostate cancer at diagnosis: findings from the prostate testing for cancer and treatment study and a systematic review. Cancer Epidemiol Biomarkers Prev. 2009;18(11):2874-2881.

41. Williams H, Powell IJ, Land SJ, et al. Vitamin D receptor gene polymorphisms and disease free survival after radical prostatectomy. Prostate. 2004;61(3):267-275.

42. Yao S, Hong CC, Bandera EV, et al. Demographic, lifestyle, and genetic determinants of circulating concentrations of 25-hydroxyvitamin D and vitamin D-binding protein in African American and European American women. Am J Clin Nutr. 2017;105(6):1362-1371.
Cancer Management and Research

\section{Publish your work in this journal}

Cancer Management and Research is an international, peer-reviewed open access journal focusing on cancer research and the optimal use of preventative and integrated treatment interventions to achieve improved outcomes, enhanced survival and quality of life for the cancer patient The manuscript management system is completely online and includes

\section{Dovepress}

a very quick and fair peer-review system, which is all easy to use. Visit http://www.dovepress.com/testimonials.php to read real quotes from published authors. 